\title{
INTERPRETATION OF OBSERVATIONS OF SPIRAL STRUCTURE IN TERMS OF THE DENSITY WAVE THEORY*
}

\author{
PER OLOF LINDBLAD \\ Stockholm Observatory, Sweden
}

\begin{abstract}
A review is given of recent theoretical and observational work on the density wave theory of spiral structure. Emphasis is put on the kinematic picture, and the question whether modern observations reveal the existence of density waves is discussed.
\end{abstract}

The most obvious phenomenon that might lead one's thoughts to a picture of density waves is a straight bar present in a differentially rotating galaxy. In the 1940's B. Lindblad (ref. Contopoulos, 1970a) developed a theory of self-sustained bar-like density waves in a galaxy with moderate differential rotation. This theory was based on the moments of the Boltzmann equation, i.e. the equation of continuity for the distribution function in six-dimensional phase space valid for a stellar system. Lin and Shu (Lin, 1966; Lin et al., 1969; Shu, 1970a, b), on the basis of the same equation of continuity, proved that a self-sustained spiral density wave was a possible solution. Their linear solution was restricted to tightly-wound spirals and small density amplitudes. This theory, as followed up by Lin, Shu, and many others, has attracted much interest because it gives quantitative relations between various structural and kinematic parameters and predicts phenomena which can be compared with observation.

The density wave theory has sometimes been criticized for being 'incomplete'. Perhaps part of this criticism is removed if we call it a density wave model of spiral galaxies, a coherent model which is in the process of being developed into a theory.

\section{The Theory of Density Waves}

In view of the development over the past two years we will approach the density wave theory from the picture of orbits in a galaxy. The foundations for this approach were laid by B. Lindblad (1958, 1964, and references given there) in the 1950's.

An orbit in the galactic plane is governed by two frequencies, both of which are functions of the distance $R$ from the centre of the galaxy:

(1) The angular frequency of circulation around the galactic centre, $\omega$.

(2) The frequency of radial oscillation, the epicyclic frequency, $\kappa$.

In general, these frequencies are not commensurable and the orbit is a nonclosed rosette. Of fundamental importance for the maintenance of large-scale density waves is the empirical fact that $\omega-\kappa / 2$ is nearly constant over the main body of our

* The density wave theory of galactic spiral structure has been reviewed several times in recent years, e.g. by Contopoulos (1970a, 1972, 1973b), and Lin (1971), and most recently in two lectures by Shu (1973). An elementary introduction to the subject has been given by Wielen (1971). 
Galaxy (Figure 1). This seems to be a general characteristic of galaxies in spite of their rather different rotation curves. The $\omega$ vs. $\kappa$ diagram for M101, for instance, looks very much the same as that in Figure 1.

For any particle or star with orbital frequencies $\omega_{\mathrm{i}}, \kappa_{\mathrm{i}}$, the orbit may always be considered as a closed ellipse with its centre at the centre of the galaxy, the ellipse rotating with the angular velocity

$$
\Omega_{\mathrm{i}}=\omega_{\mathrm{i}}-\frac{\kappa_{\mathrm{i}}}{2} \text {. }
$$

The particles travel in the forward direction around the orbit with respect to its rotating apsidal line. If $\omega-\kappa / 2$ is constant over some part of the system, this means

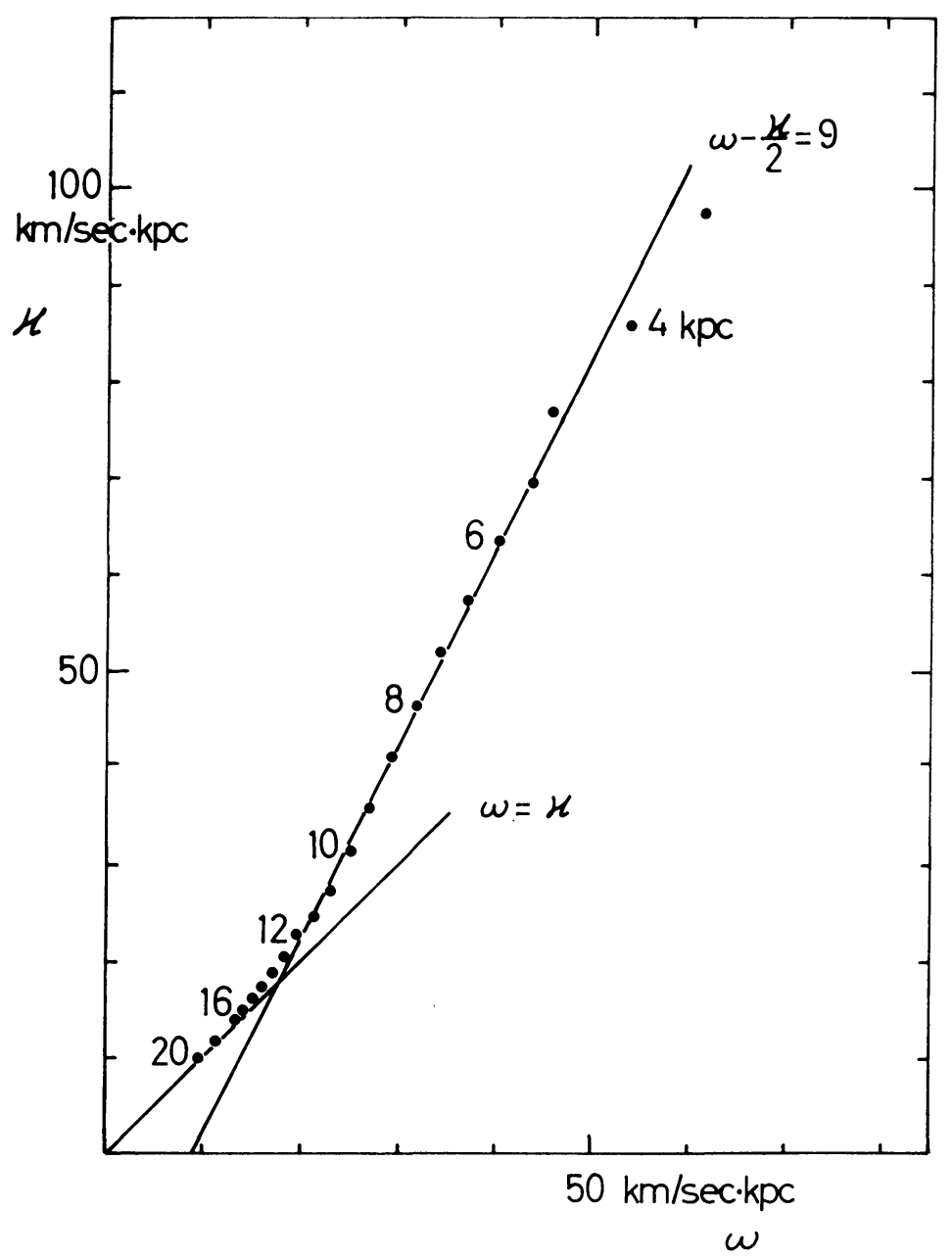

Fig. 1. Relation between the frequencies (") and $k$ for the Galaxy. Distances from the centre in $\mathrm{kpc}$ are indicated. 
that all such orbital ellipses turn with the same speed. This also means that a cloud would disperse along such a closed elliptic orbit. The resulting closed massive ring is generally called a dispersion ring. Another consequence would be that any one-sided density asymmetry will be drawn out into a bi-symmetrical one. This would be the basic reason for the bi-symmetrical character of two-armed spiral and barred type galaxies.

The nearly constant value of $\omega-\kappa / 2$ means that, if we arrange a set of orbits into a spiral pattern for instance (Figure 2, due to Kalnajs, 1973), this arrangement will be preserved for some time and will turn with the pattern velocity $\omega-\kappa / 2$. The stars in their circulation will move almost tangentially to the arm within the arm

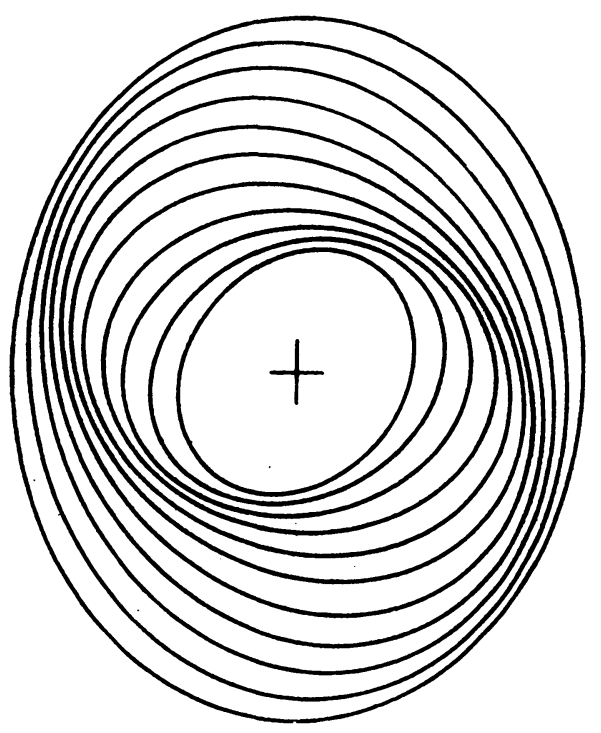

Fig. 2. A set of closed elliptical orbits arranged into a spiral pattern (after Kalnajs, 1973).

pattern and there have their maximum inward motion if the spiral arms are trailing. If mass is added to the orbits, the pattern velocity will be slightly higher than $\omega-\kappa / 2$ as given for an unperturbed orbit. What Lin showed is that, if you give mass to these orbits, you can for some time have a self-supporting spiral density wave with a constant pattern velocity.

If the pattern velocity, $\Omega_{\mathrm{p}}$, is larger than some mean value $\langle\omega-\kappa / 2\rangle$, we will in general have three basic resonance regions for different values of $R$ :

(1) The co-rotation region (particle resonance), where $\omega=\Omega_{\mathrm{p}}$.

(2) The inner resonance region, where $\omega-\kappa / 2=\Omega_{\mathrm{p}}$. The unperturbed closed elliptic orbit here turns with the speed of the wave.

(3) The outer resonance region, where $\omega+\kappa / 2=\Omega_{\mathrm{p}}$. An unperturbed closed elliptic orbit with the particles moving in a retrograde direction around the orbit with respect to its apsidal line here turns with the speed of the wave. 
We may ask why a galaxy should arrange itself as shown in Figure 2. One reason, suggested by Lynden-Bell and Kalnajs (1972), is that a flat differentially-rotating galaxy with given angular momentum can lower its rotational energy (or increase its entropy) by transporting angular momentum outwards; only in trailing spiral structures do the gravity torques carry angular momentum outwards.

An important contribution to the density wave picture are the computations by Roberts (1969), and more recently by Shu et al. (1973), of the gas flow in this spiral gravitational field and the formation of shock waves. In Kalnajs' presentation the shock phenomena arise in the interstellar gas when the dispersion orbits have been turned to such a degree that they intersect (Figure 3). Galactic shocks arise for waves of sufficiently large amplitude, i.e. for sufficiently large eccentricity of the rings.

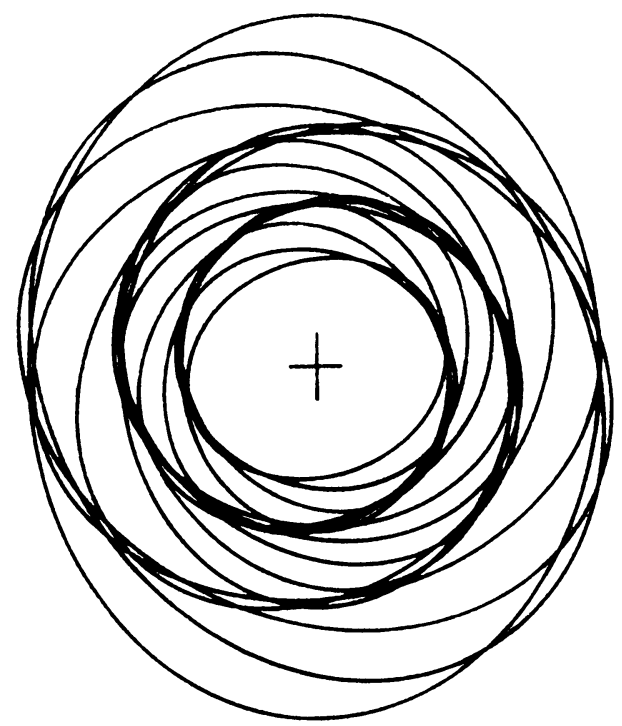

Fig. 3. The amplitude of the elliptical motion increased (when compared to the wavelength of spiral structure) until a galactic shock occurs (after Kalnajs, 1973).

Specifically, Shu et al. predict that broad arms with open spiral structure should prevail in galaxies with weak differential rotation, whereas narrow arms and tightly wound spiral structure should prevail in galaxies with strong differential rotation. They also claim that a number of special resonances, in addition to the principal ones mentioned above, can give rise to secondary compressions of the gas.

The galactic shocks would be the location of the most intense star formation. Of great interest in this respect is the work by Shu et al. (1972) on galactic shocks in an interstellar medium with two stable phases in pressure equilibrium. The non-linear wave pattern just described is followed by the hot intercloud phase which then compresses the cold clouds to high densities in the shock region.

To be sure, density waves will propagate from co-rotation to the inner resonance region (Toomre, 1969; Shu, 1970b), and galactic shocks will be damped (Kalnajs, 
1972; Roberts and Shu, 1972) in a time span which in both cases may be of the order of $10^{9} \mathrm{yr}$. The three principal resonance regions have been considered as sources and sinks for angular momentum and wave energy. Partly for this reason interest has been taken in the last few years in resonance effects, and the resonance regions have been interpreted as key regions for the creation and maintenance of density waves.

Perturbed orbits around the resonance regions have been studied by Contopoulos (1970b) and Vandervoort (1973). As the inner resonance region is approached, they find increasing eccentricity of stable periodic orbits and surrounding tube orbits, which could give the inner region the appearance of a bar-like structure. Near the resonance a second family of elongated stable periodic orbits, roughly perpendicular to the first, appears. If one proceeds inside the resonance, the first set of tube orbits shrinks and eventually disappears, while the periodic orbits of the second family become nearly circular.

Contopoulos (1973a) has further studied resonance effects at co-rotation. He finds four equilibrium points, two of which are stable and situated at spiral density minima, and he argues that large masses could be trapped around these points. Feldman and Lin (1973, see also Feldman 1973) claim that a rigidly-rotating, weakly bar-like structure in the central regions of a galaxy forces a tightly-wound trailing spiral wave at co-rotation. This wave is then supposed to propagate towards the inner resonance region.

Thus, we are given a fairly detailed density-wave model for relatively tightly wound spiral galaxies. The perturbing action is caused by a rigidly-rotating smooth spiral stellar wave with a density contrast of a few per cent and with systematic deviations from circular velocities of a few tens of kilometres per second; it is thus very difficult to observe. On top of these waves rides, like foam on the waves of the ocean, the interstellar gas compressed by shocks and with this the radio and optical spiral tracers. The density of wave energy is comparable to the densities contained locally in turbulent gas motions, starlight, cosmic-ray particles and interstellar magnetic fields (Shu, 1973), thus we can expect that even if the grand design is regular the local spiral structure may be somewhat disordered.

\section{Observation of Density Waves}

\subsection{THE GaLAXY}

The picture presented above has, of course, evolved through confrontation with observations. For observers it is an appealing working hypothesis and we would like to confront it with the present observing material. Do we see density waves?

The density wave theory has recently been criticized on observational grounds by Piddington (1973a, b). However, one of the main points made by Piddington - that the interstellar gas does not form strings along the spiral arms - now seems to be definitely refuted by the beautiful observations just shown by Professor Oort (p. 375).

Thus, we have good reason to believe that the narrow ridges we find in the velocity distribution of neutral hydrogen as a function of longitude in our Galaxy correspond 
to a spiral structure of hydrogen arms. A difficulty when comparing the density wave theory with observations is that the linear theory does not give a unique value for the pattern velocity unless we already know the shape of the pattern. To try to fit a density wave to a spiral pattern found by the assumption of purely circular motions has its dangers. This is clearly brought out by the interesting attempt to apply density wave theory, including large scale shock formation, to our Galaxy, made by Roberts (1972) for a region of the Perseus arm. In Figure 4 we see how a line profile, which interpreted by an assumption of circular motion would give a complicated density pattern, can be represented by a single spiral wave, where peaks in the velocity distribution arise partly from a shock and partly from velocity crowding over intervals where the radial velocity changes little with distance. Thus, the results based on the assumption of circular motion may not give even a first approximation to the true structure and there is the consequential necessity of a complete re-evaluation of galactic hydrogen observations in terms of the spiral shock theory - a tremendous but necessary job to be done. Verschuur (1973) has criticized this approach, but I
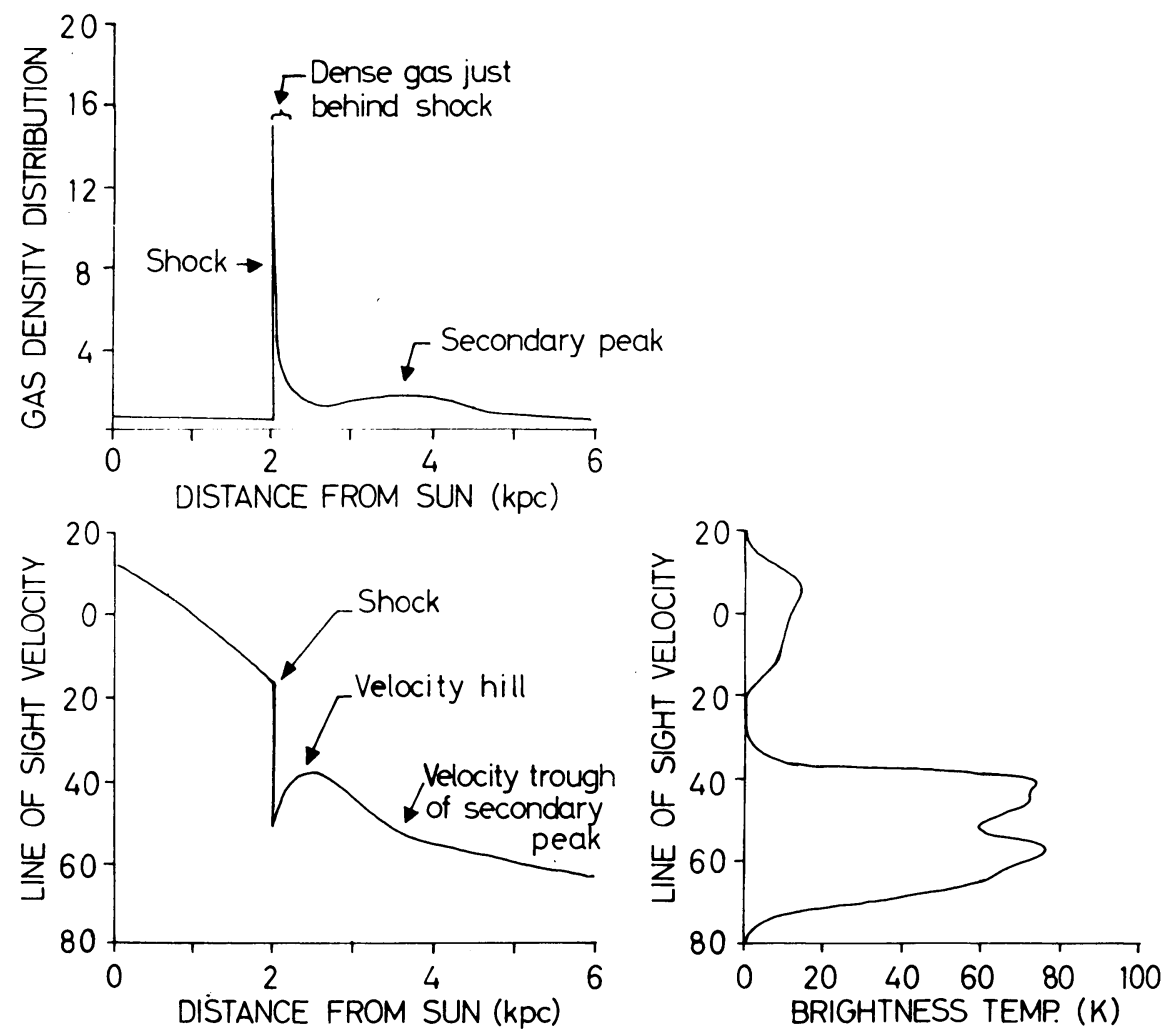

Fig. 4. Theoretical features of a two-armed spiral shock model in the longitude range $130^{\circ}-140^{\circ}$. Upper left: Gas density distribution (unit = average density) as a function of distance from the Sun. The basic motion of the gas is from left to right. Lower left: Line-of-sight velocities versus distance from the Sun. Lower right: Resulting theoretical line profile (after Roberts, 1972). 
don't think that his argument solves the problem. Anyhow, confrontation with the observations in the Galaxy, as they are now available in various surveys, emphasizes the need to work out the density-shock wave theory in three dimensions. A first attempt to do this has been made by Tosa (1973).

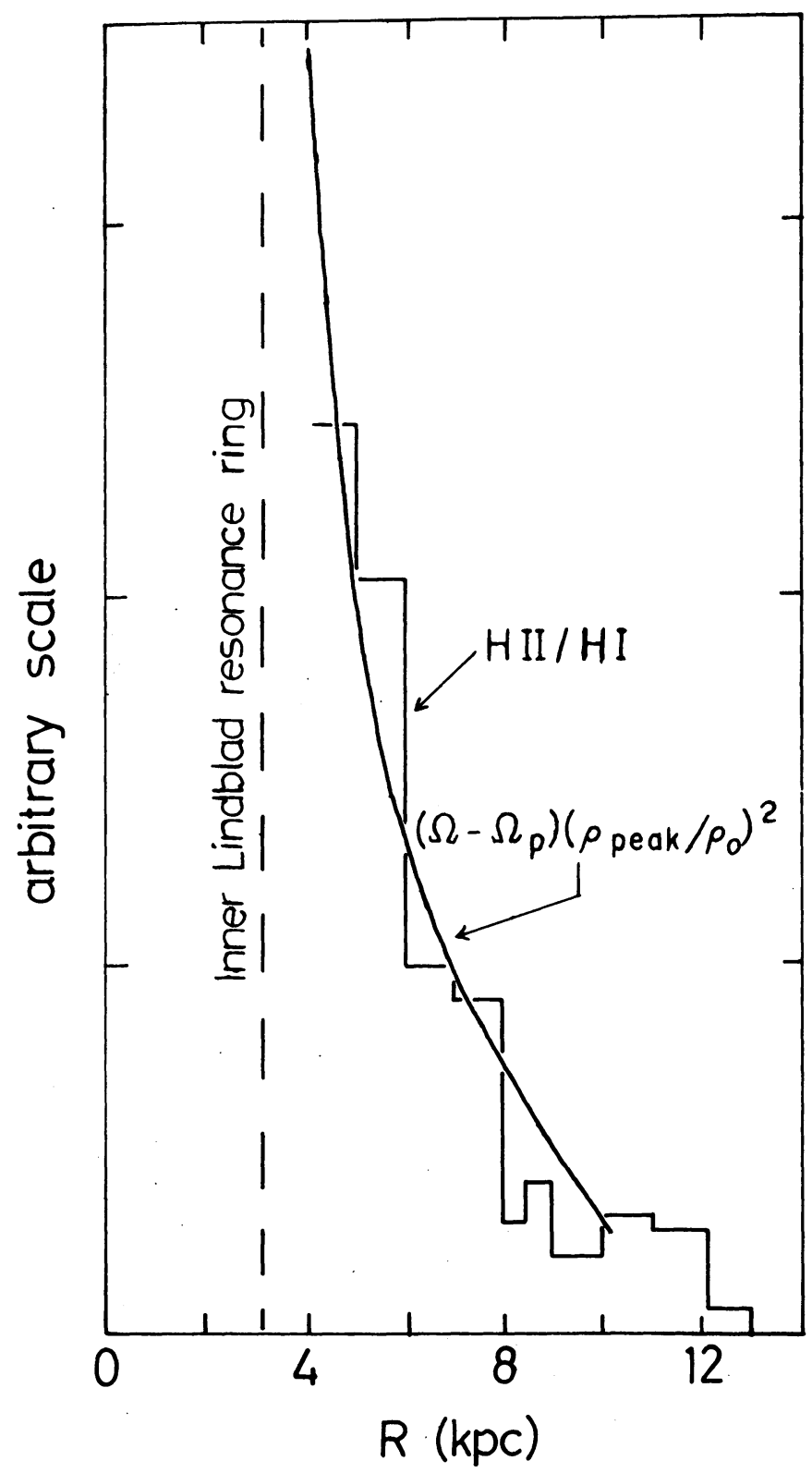

Fig. 5. The abundance of $\mathrm{H}_{\text {II }}$ relative to that of $\mathrm{H}_{\mathrm{I}}$ in the Galaxy as compared to a theoretical relation for the frequency of compression times the square of the peak compression strength in a galactic shock (after Shu, 1973). (For $\left(\Omega-\Omega_{p}\right)$ read $\left(\omega-\Omega_{p}\right)$, Editor.) 
An important observational phenomenon in our Galaxy, which also seems to have its counterpart in other galaxies, is the discrepancy between the distribution of $\mathrm{H}$ II and $\mathrm{H}$ I which indicates a difference between the rate of star-formation and average hydrogen density. This has just been discussed by Professor Oort (p. 375). In view of the density wave theory, the rapid increase inward of the ratio $\mathrm{H} \mathrm{II} / \mathrm{H} \mathrm{I}$ can be attributed to two causes (Shu, 1973): the increase inward of the frequency $\left(\omega-\Omega_{\mathrm{p}}\right)$ at which the interstellar gas is periodically compressed, and the increase inward of the peak compression $\varrho_{\text {peak }} / \varrho_{0}$. Shu (Figure 5) demonstrates the close resemblance between the run of $\mathrm{HII} / \mathrm{H}$ I and the quantity $\left(\omega-\Omega_{\mathrm{p}}\right)\left(\varrho_{\text {peak }} / \varrho_{0}\right)^{2}$.

The rotation curve of the Galaxy is such that there should exist an inner resonance region for a two-armed spiral wave at about 3-4 kpc from the centre. Attempts to explain the ' $3-\mathrm{kpc}$ arm' and other apparently expanding features in the vicinity of that region in terms of a dispersion ring have been made by Shane (1972) and by Simonson and Mader (1973).

\subsection{EXTERnAL GalaXieS}

The external galaxy that has been most thoroughly investigated for comparison with the density wave theory is M51. Well known to everyone are the beautiful results obtained by Mathewson et al. (1972), using the Westerbork synthesis telescope to map the $20-\mathrm{cm}$ radio continuum due to synchrotron radiation. A striking feature is the two radio arms. The fact that these radio arms coincide with the dust lanes on the inner side of the optical arms is strong support for the spiral shock-wave theory, the radio emission being due to the compression of the magnetic field along with the gas and the increase in the number density of relativistic electrons.

A very thorough analysis based on optical observations with a Fabry-Pérot interferometer has been carried out by Tully (1974) who finds streaming motions in the region of the inner spiral arms consistent with the density wave theory. From the amplitude of the streaming motions, he obtains the very high value of $15-20 \%$ for the ratio of the spiral potential to the axisymmetric potential and, from the inclination of the dust lanes, a rather high pattern velocity which places co-rotation only $2.4 \mathrm{kpc}$ from the centre. An analysis of the non-circular motions inside the inner resonance region shows that they can best be represented by motions of the dispersion-orbit type, which apparently supports Contopoulos' calculations. In accordance with Toomre and Toomre (1972), Tully suggests that the outer spiral structure arises from the tidal effects of NGC 5195, which then would cause the density wave to propagate inwards from co-rotation. Also, Weliachew and Gottesman (1973) find from $21-\mathrm{cm}$ studies that the velocities in the north-eastern part of the galaxy agree with the predictions of the Toomres. In view of the rather elongated orbit of NGC 5195 in the Toomres' model, this would mean that we happen to see M51 in a stage of strong arm formation. Although there are several arguments supporting this view, it still means that we have to apply two different mechanisms to explain spiral arms with shocks delineated by dark lanes in different parts of the galaxy. Van der Kruit (1973), on the other hand, finds that NGC 4321 and M51 show a similar structure in the radio 
continuum and draws the conclusion that the structure of M51 can only in a minor way be the result of tidal action from NGC 5195.

Thus, it seems very desirable to confirm Tully's analysis with the new $21-\mathrm{cm}$ line measurements which have been carried out with the Westerbork telescope and are being analyzed by Shane and Dekker at Leiden. The total hydrogen map (Figure 1 in the article by Professor Oort, p. 375) shows clearly how the hydrogen follows the

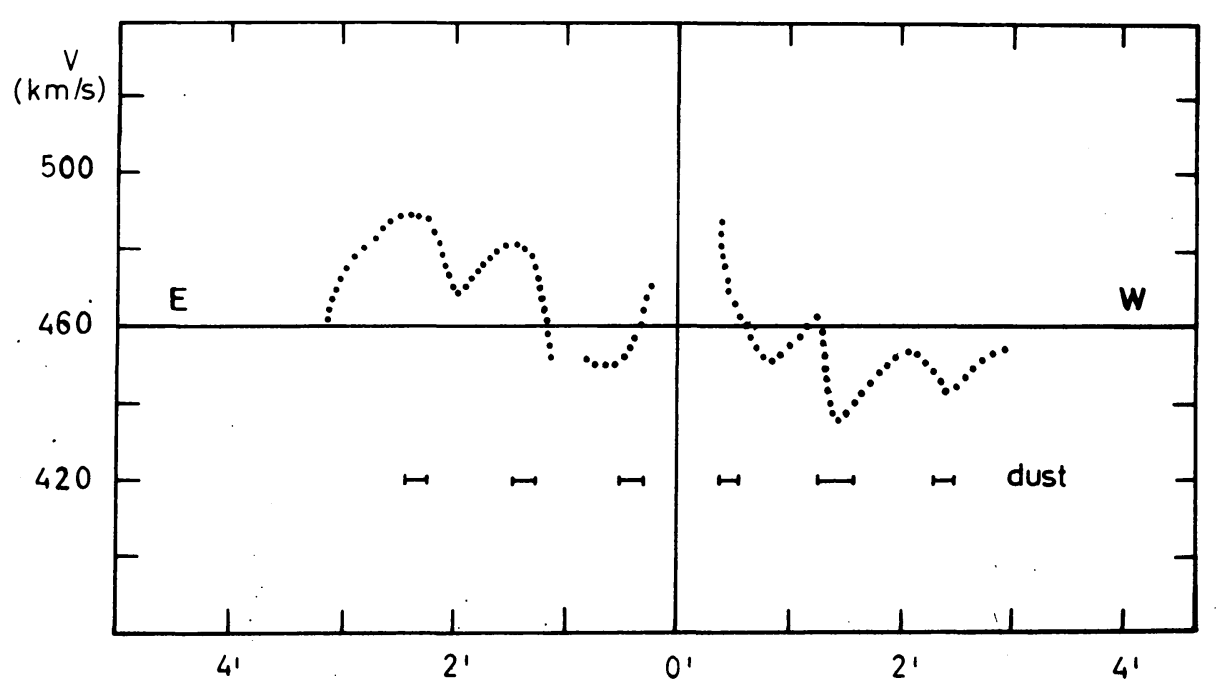

Fig. 6. Radial motion of neutral hydrogen along the minor axis of M51 as a function of distance from the centre. The position of dust lanes are indicated (courtesy W. W. Shane and E. Dekker).

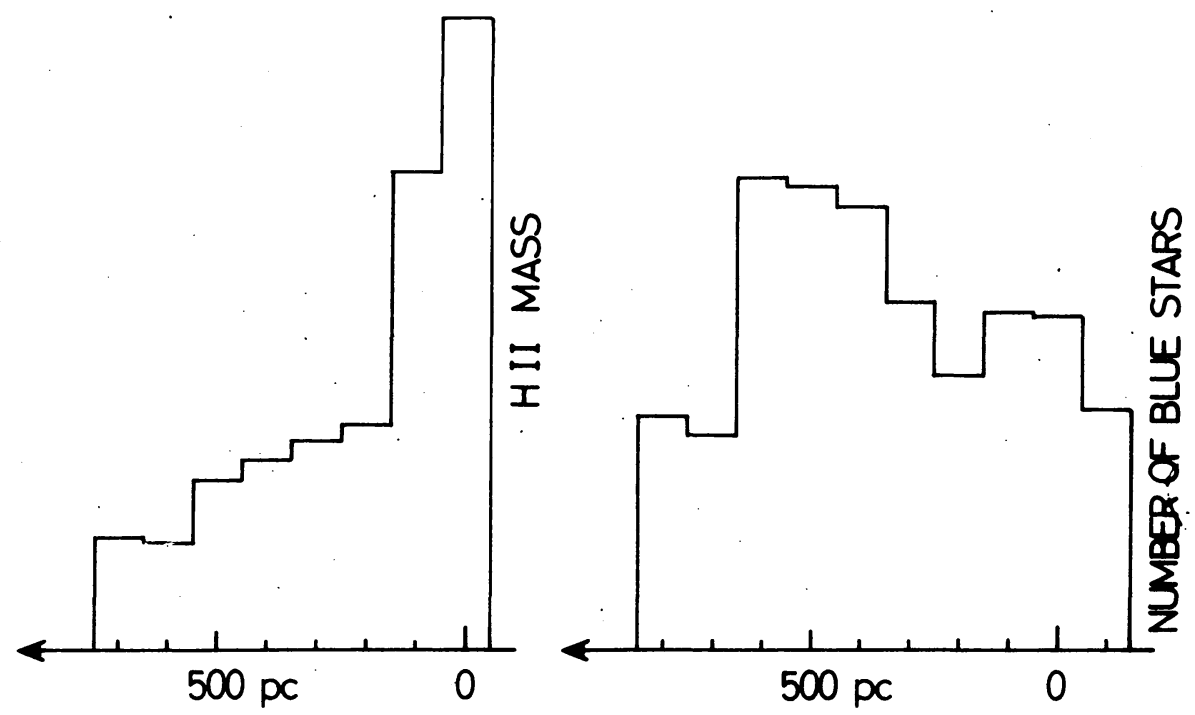

Fig. 7. Distribution of ionized hydrogen (left) and blue stars (right) as a function of distance from the following edge of a spiral arm in M33 (after Courtès and Dubout-Crillon, 1971). 
optical arms. Also, the maximum hydrogen line intensity has a tendency to fall on the dust lanes, where the shock is supposed to be. However, according to Shane (private communication) the hydrogen maximum could very well be shifted with respect to the shock-wave, partly because the clouds in the shock may be optically thick with high self-absorption and partly because of the limited angular resolution which averages over the shock and the following density shoulder. The velocity field derived so far is only preliminary, but it seems that wave-type motions may be present with maximal inward motions coinciding with the dust arms (Figure 6), in agreement with the shock-wave picture.

M81 is an interesting case because of the regular spiral structure and the beautiful hydrogen arms. Here also the hydrogen arms seem to fall right on top of the optical arms (Rots and Shane, 1974; see also p. 378). A very preliminary hydrogen velocity

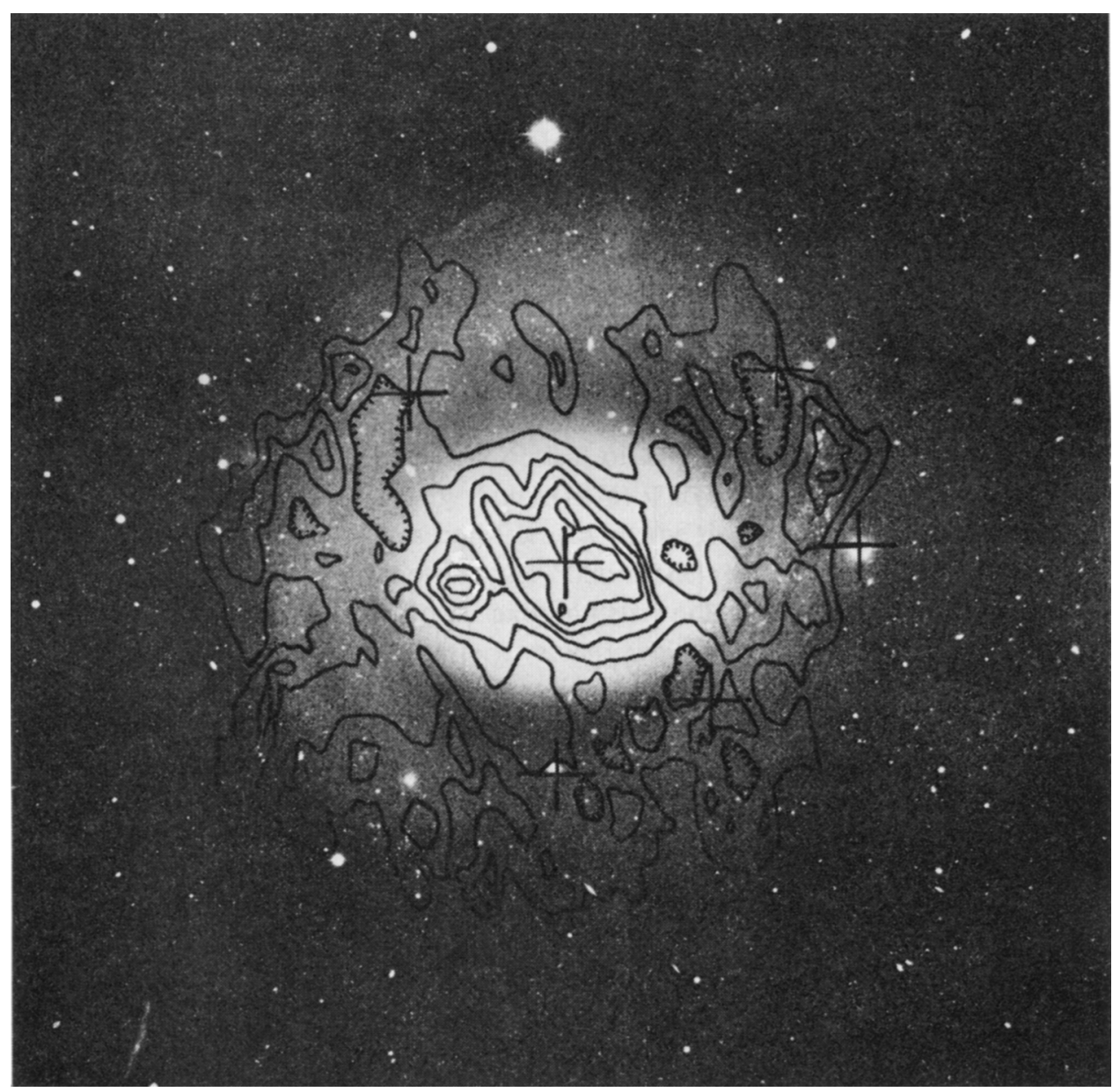

Fig. 8. Total neutral hydrogen distribution in M94 as observed with the Westerbork telescope (Bosma et al., 1974) 
picture shows indication of waves, although the question of symmetry is not quite clear. A final picture is being produced by Rots at the Kapteyn Laboratory.

M33 has been observed in the 21-cm hydrogen line by Wright et al. (1972, Warner et al., 1973) and again the integrated hydrogen is correlated with the optical spiral arms. The authors failed to detect any large-scale density wave with an amplitude greater than $3 \mathrm{~km} \mathrm{~s}^{-1}$. Courtès and Dubout-Crillon (1971) have studied a part of the southern arm of M33 using interference and coloured filters and have determined the relative distributions of $\mathbf{H}$ il regions and bright stars across the spiral arm (Figure 7). If the gravitational force is dominant their result is a strong indication of wave motion. According to Roberts the pattern velocity would then be slower than the circular velocity and the co-rotation point would lie further out than the region studied. Dixon (1971) has investigated the same arm further out by $B, V$ photometry. He finds

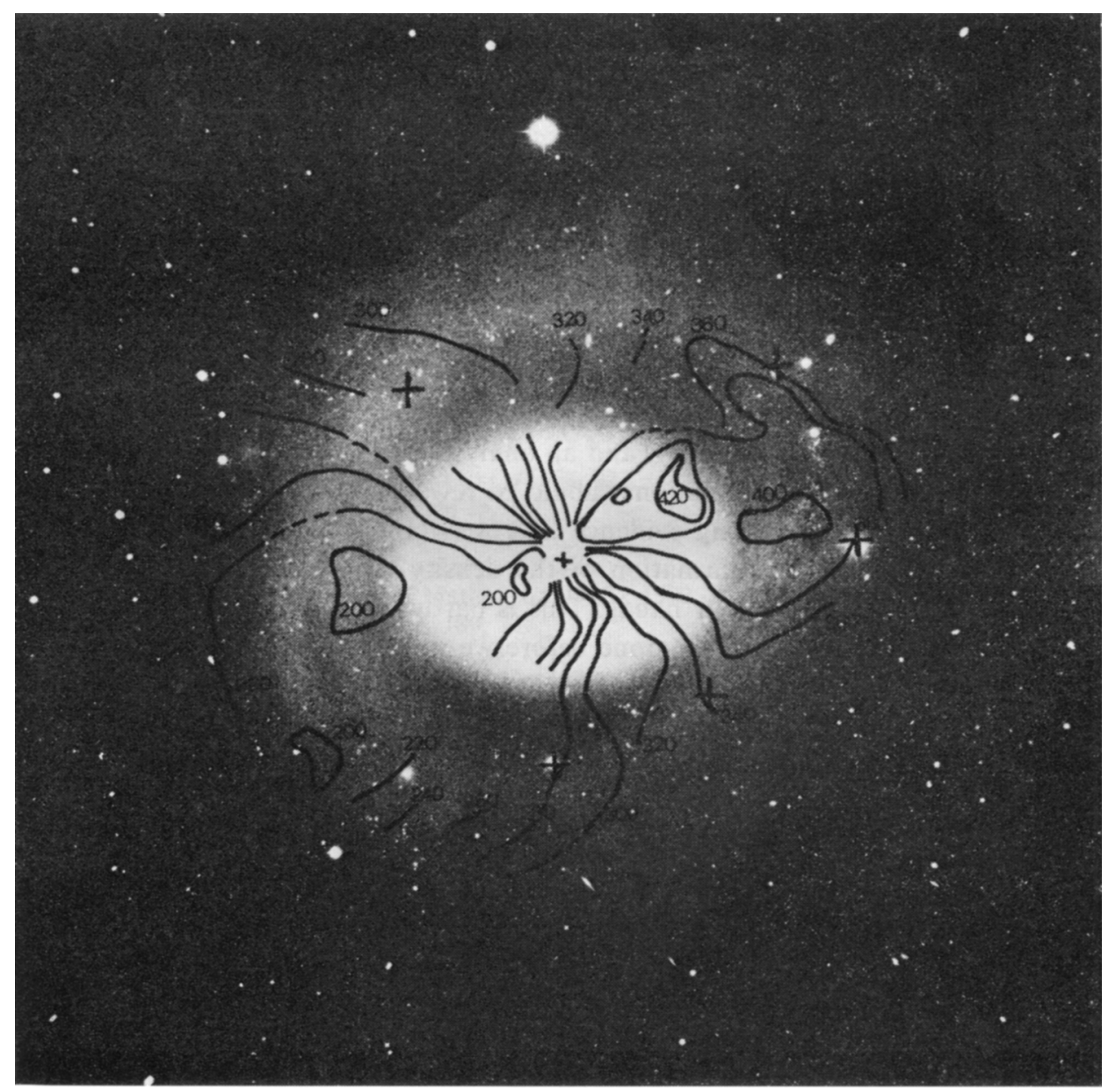

Fig. 9. Velocity field of neutral hydrogen in M94 as observed with the Westerbork telescope (Bosma et al., 1974) 
an age progression of stars across the spiral arm opposite to that of Courtès and Dubout-Crillon. In Roberts' picture this would indicate a co-rotation distance somewhere between the radii of the two surveys, i.e. about $3 \mathrm{kpc}$ from the centre, giving a relatively high pattern velocity also in this system.

The case of NGC 4258 (van der Kruit et al., 1972) has been presented by Professor Oort, who suggested that outflow of matter from the nucleus leads to a reformation of the spiral structure. This would not concern the basic density wave in the stellar disk which is, presumably, little perturbed by the expulsion of gas from the nucleus. The outflowing matter could perhaps be collected in the spiral shocks eventually and thus add to the material tracing the spiral structure.

It would be of the outmost importance for our understanding of density waves if we could identify the different resonance regions in the galaxies observed. It is interesting in this connection to look at the galaxy M94 (NGC 4736), where an inner ring structure was discussed in the paper by van der Kruit (this volume, p. 431). This rather tightly wound spiral also has an extensive outer ring which, according to recent observations with the Westerbork telescope (Bosma et al., 1974), is clearly outlined by the neutral hydrogen (Figure 8). The ring seems to be attached at two points to the inner region, but its connection with the inner spiral structure is difficult to establish. The preliminary velocity field given in Figure 9 seems to show a discontinuity across the gap between the outer ring and the main body. It has been suggested (P.O. Lindblad, 1960) that, in the case of M94, this gap corresponds to the co-rotation region. The numerical many-body computations on which this suggestion was based may also to some extent illustrate the theoretical discussions of the co-rotation region by Contopoulos, and by Feldman and Lin, mentioned above. The kinematics of this galaxy seem to be worth a detailed study, and an analysis combined with a theoretical discussion and numerical computations should be rewarding.

In summary, there is strong evidence that we observe density waves in galaxies, although a quantitative confirmation of the density wave theory, or some variant of the theory, is not yet in our hands. This is partly due to the state of the theory, where much work still has to be done before we get a complete picture, and partly to that of the observations, where the really relevant data have only just begun to come in.

With the present vigorous activity and rapid development on both the theoretical and observational side there is promise that we will learn many new interesting facts in the near future.

\section{References}

Bosma, A., van de Hulst, J. M., and Sullivan, W. T.: 1974 (in preparation).

Contopoulos, G.: 1970a, in W. Becker and G. Contopoulos (eds.), 'The Spiral Structure of Our Galaxy', IAU Symp. 38, 303.

Contopoulos, G.: 1970b, Astrophys. J. 160, 113.

Contopoulos, G.: 1972, The Dynamics of Spiral Structure, Lecture Notes, Astronomy Program, Univ. of Maryland.

Contopoulos, G.: 1973a, Astrophys. J. 181, 657. 
Contopoulos, G.: 1973b, The Density Wave Theory of Spiral Structure, in Proceedings of the Advanced Study Institute on the Dynamical Structure and Evolution of Stellar Systems, Saas-Fee (Publ. Geneva Obs.)

Courtès, G. and Dubout-Crillon, R.: 1971, Astron. Astrophys. 11, 468.

Dixon, M. E.: 1971, Astrophys. J. 164, 411.

Feldman, S. I.: 1973, Ph.D. Thesis, MIT.

Feldman, S. I. and Lin, C. C.: 1973, Studies in Appl. Math. 52, 1.

Kalnajs, A. J.: 1972, Astrophys. Letters 11, 41.

Kalnajs, A. J.: 1973, Proc. Astron. Soc. Australia 2, 173.

Lin, C. C.: 1966, J. SIAM Appl. Math. 14, 876.

Lin, C. C.: 1971, in C. de Jager (ed.), Highlights of Astronomy 2, 88.

Lin, C. C., Yuan, C., and Shu, F. H.: 1969, Astrophys. J. 155, 721.

Lindblad, B.: 1958, Stockholm Obs. Ann. 20, No. 6.

Lindblad, B.: 1964, Astrophys. Norv. 9, 103 (= Stockholm Obs. Medd. No. 145).

Lindblad, P. O.: 1960, Stockholm Obs. Ann. 21, No. 4.

Lynden-Bell, D. and Kalnajs, A. J.: 1972, Monthly Notices Roy. Astron. Soc. 157, 1.

Mathewson, D. S., van der Kruit, P. C., and Brouw, W. N.: 1972, Astron. Astrophys. 17, 468.

Piddington, J. H.: 1973a, Astrophys. J. 179, 755.

Piddington, J. H.: 1973b, Monthly Notices Roy. Astron. Soc. 162, 73.

Roberts, W. W.: 1969, Astrophys. J. 158, 123.

Roberts, W. W.: 1972, Astrophys. J. 173, 259.

Roberts, W. W. and Shu, F. H.: 1972, Astrophys. Letters 12, 49.

Rots, A..H. and Shane, W. W.: 1974, Astron. Astrophys. 31, 245.

Shane, W. W.: 1972, Astron. Astrophys. 16, 118.

Shu, F. H.: 1970a, Astrophys. J. 160, 8S.

Shu, F. H.: 1970b, Astrophys. J. 160, 99.

Shu, F. H.: 1973, Lectures delivered at the Advanced Study Institute on the Interstellar Medium, Schliersee, preprint.

Shu, F. H., Milione, V., Gebel, W., Yuan, C., Goldsmith, D. W., and Roberts, W. W.: 1972, Astrophys. J. 173, 557.

Shu, F. H., Milione, V., and Roberts, W. W.: 1973, Astrophys. J. 183, 819.

Simonson, S. C. and Mader, G. L.: 1973, Astron. Astrophys. 27, 337.

Toomre, A.: 1969, Astrophys. J. 158, 899.

Toomre, A. and Toomre, J.: 1972, Astrophys. J. 178, 623.

Tosa, M.: 1973, Publ. Astron. Soc. Japan, 25, 191

Tully, R. B.: 1974, Astrophys. J. Suppl. 27, 415.

van der Kruit, P. C.: 1973, Astron. Astrophys. 29, 249.

van der Kruit, P. C., Oort, J. H., and Mathewson, D. S.: 1972, Astron. Astrophys. 21, 169.

Vandervoort, P. O.: 1973, Astrophys. J. 180, 739.

Verschuur, G. L.: 1973, Astron. Astrophys. 24, 193.

Warner, P. J., Wright, M. C. H., and Baldwin, J. E.: 1973, Monthly Notices Roy. Astron. Soc. 163, 163. Weliachew, L. and Gottesman, S. T.: 1973, Astron. Astrophys. 24, 59.

Wielen, R.: 1971, Mitt. Astron. Ges. 30, 31.

Wright, M. C. H., Warner, P. J., and Baldwin, J. E.: 1972, Monthly Notices Roy. Astron. Soc. 155, 337.

\section{DISCUSSION}

G. de Vaucouleurs: The nature of the outer rings varies a little with the Hubble classification of the inner part; in some galaxies, such as NGC 1068 and NGC 1291, two faint spiral arms emerge from the outer ring and the ring itself is made up of many short spiral arcs.

Lindblad: Such observations should throw light upon how arms are excited at co-rotation.

G. de Vaucouleurs: It is an interesting empirical fact that many of the galaxies which have a central ring-structure, such as M94 (NGC 4736), NGC 4221, 6753 and 7217, also have a large outer ring.

Allen: Some months ago, Piddington (Astrophys. J. 179, 755, 1973) asserted that there was no evidence in the then available data to support the widely-held belief that galaxian spiral arms consist, among other things, of elongated, connected concentrations of neutral hydrogen. Piddington used 
the radio synthesis data of Rogstad and Shostak (Astron. Astrophys. 13, 99, 1971) to support his claim that the maxima in the $\mathrm{H}$ I distribution of M101 did not fall on the obvious optical spiral arms. The higher angular resolution of the Westerbork observations which we have presented (p. 425) (a factor of 9 increase in linear resolution over the observations by Rogstad and Shostak) reveals a very good general correspondence of small-scale features in the $\mathrm{H}$ I with the optical spiral structure except in the central regions of the galaxy, where these $\mathrm{H}$ I features are almost absent.

I also draw attention to Shu's study (Astron. Astrophys., in press) of the enhancement of synchrotron emission by compression of interstellar gas and magnetic fields. Because of the Parker instability, which causes compressed field to 'pop' out of the galactic plane, Shu finds that the synchrotron emissivity goes as the first power of the density compression, rather than the third power as many have assumed.

Lindblad: Similar results have been derived by Tosa.

Oort: The compression would depend upon how fast the gas cools, and this would depend upon the initial density.

Allen: Yes indeed. 